\title{
On the Topology of Isochronous Centers of Hamiltonian Differential Systems
}

\author{
Guangfeng Dong* \\ Department of Mathematics, Jinan University, \\ Guangzhou 510632, P. R. China \\ donggf@jnu.edu.cn \\ Changjian Liu \\ School of Mathematics (Zhuhai), Sun Yat-Sen University, \\ Zhuhai 519082, P. R. China \\ liuchangj@mail.sysu.edu.cn \\ Jiazhong Yang \\ School of Mathematical Sciences, Peking University, \\ Beijing 100871, P. R. China \\ jyang@math.pku.edu.cn
}

Received January 16, 2019

\begin{abstract}
In this paper, we study the topology of isochronous centers of Hamiltonian differential systems with polynomial Hamiltonian functions $H(x, y)$ such that the isochronous center lies on the level curve $H(x, y)=0$. We prove that, in the one-dimensional homology group of the Riemann surface (removing the points at infinity) of level curve $H(x, y)=h$, the vanishing cycle of an isochronous center cannot belong to a subgroup generated by those small loops such that each of them is centered at a removed point at infinity of having one of the two special types described in the paper, where $h$ is sufficiently close to 0 . Besides, we present some topological properties of isochronous centers for a large class of Hamiltonian systems of degree $n$, whose homogeneous parts of degree $n$ contain factors with multiplicity of no more than $n / 2$. As applications, we study the nonisochronicity for some Hamiltonian systems with quite complicated forms which are usually very hard to handle by the classical tools.
\end{abstract}

Keywords: Isochronous center; Hamiltonian systems; vanishing cycle.

\section{Introduction and Main Results}

Isochronous center is one of the most interesting singularities of planar integrable differential systems and has been studied extensively for decades (see, e.g. Chavarriga \& Sabatini. 1999: Fernandes et al. 2017: Han \& Romanovski. 2012: Llibre \& Valls, 2011] and references therein), especially for
Hamiltonian differential systems. Consider the following Hamiltonian differential system on $\mathbb{R}^{2}$

$$
\dot{x}=\frac{\partial H}{\partial y}, \quad \dot{y}=-\frac{\partial H}{\partial x}, \quad(x, y) \in \mathbb{R}^{2},
$$

where the Hamiltonian function $H(x, y)=\left(x^{2}+\right.$ $\left.y^{2}\right) / 2+\sum_{i+j=3}^{n} a_{i j} x^{i} y^{j}$ is a polynomial of degree $n$

\footnotetext{
*Author for correspondence

This is an Open Access article published by World Scientific Publishing Company. It is distributed under the terms of the Creative Commons Attribution 4.0 (CC-BY) License. Further distribution of this work is permitted, provided the original work is properly cited.
} 
in $\mathbb{R}[x, y]$, and the origin is an isolated singularity of center type. The period function of a center is defined through the period of each periodic orbit inside the period annulus of the center. Clearly it is well defined. If the period function is constant, then the center is conventionally called isochronous.

The isochronicity of Hamiltonian systems, as a special case within the more general category, is already a very subtle problem and has been characterized completely only for very few families. It is proved in Chicone \& Jacobs, 1989] that in the potential case, the unique polynomial isochronous center is the linear one. When the Hamiltonian function takes the form $H(x, y)=F(x)+G(y)$, it is proved in Cima et al., 2000] that the unique isochronous center turns out to be the linear one, too. In Cima et al., 1999], the polynomial Hamiltonian isochronous centers for $n=4$ are investigated with a corresponding classification. On the other hand, it has been shown, see, for example, Christopher \& Devlin, 1997; Gasull et al., 1997], that Hamiltonian systems have no isochronous centers if they have homogeneous nonlinearities. Some other related results can be found in, e.g. [Llibre \& Romanovski, 2015] and the references therein.

The story of isochronicity becomes more interesting as the plots go on. Everything seems reasonable when it is proved in the work of Loud (see Loud, 1964]) that, for $n=3$, no polynomial Hamiltonian systems admit isochronous centers. It is also understandable that the authors of JJarque \& Villadelprat, 2002 proved that every center of a planar polynomial Hamiltonian system for $n=5$ is nonisochronous. It is not surprising to conjecture in Jarque \& Villadelprat, 2002] that planar polynomial Hamiltonian systems with odd number $n$ have no isochronous centers. Indeed, some results (e.g. in Chen et al., 2008; Mañosas \& Villadelprat, 2002; Wang et al., 2010]) for systems (1) on $\mathbb{R}^{2}$ support this conjecture. However, the examples given in Gavrilov, 1997] show that the above conjecture is false when the concept of isochronous center is generalized to systems on $\mathbb{C}^{2}$, i.e. the Hamiltonian function $H(x, y) \in \mathbb{C}[x, y]$.

Let us first explain this point of view. Without loss of generality we assume the following Hamiltonian system

$$
\frac{\mathrm{d} x}{\mathrm{~d} t}=\frac{\partial H}{\partial y}, \quad \frac{\mathrm{d} y}{\mathrm{~d} t}=-\frac{\partial H}{\partial x}, \quad(x, y) \in \mathbb{C}^{2}, \quad t \in \mathbb{C},
$$

has only the isolated singularities and the origin is a singularity of Morse type, where the Hamiltonian function $H(x, y)=\sum_{k} H_{k}(x, y)=$ $\sum_{i+j=2}^{n} a_{i j} x^{i} y^{j} \in \mathbb{C}[x, y]$. Then the topology of nonsingular affine algebraic curve $C_{h}$ defined by $H(x, y)-h=0$ is a genus $g$ sphere with some removed points at infinity for $h$ sufficiently close to 0 but $h \neq 0$. One can associate a corresponding vanishing cycle $\gamma_{h}$ to the critical value $h=0$, which is a one-dimensional cycle vanishing at $h=0$ in the one-dimensional homology group $\mathcal{H}_{1}\left(C_{h}, \mathbb{Z}\right)$ and can be characterized by the following purely topological property: modulo orientation and the free homotopy deformation on $C_{h}$, as $h \rightarrow 0$, the cycle $\gamma_{h}$ can be represented by a continuous family of loops on $C_{h}$ of lengths that tend to zero. This description explains the terminology. Respectively $T(h)=\oint_{\gamma_{h}} \mathrm{~d} t$ is called a period of system (2). If $T(h)$ is a nonzero constant independent of $h$ for $h \neq 0$, then the origin is called generalized isochronous. If $(x, y) \in \mathbb{R}^{2}$ and $t \in \mathbb{R}$, this definition coincides with the classical isochronous center.

In the complex setting, a basic problem is to describe which element the vanishing cycle of an isochronous center represents in the homology group of $C_{h}$. It is closely related to the properties of points at infinity on $C_{h}$. In reference Gavrilov, 1997], the author proved that the vanishing cycle of an Morse isochronous center is homologous to a zero cycle on the Riemann surface of $C_{h}$, under certain assumptions on the Hamiltonian function $H(x, y)$. In this paper, we will study the properties of a quite large class of points at infinity and illustrate the relationship between them and an isochronous center.

Consider the corresponding projective closure $\bar{C}_{h}$ of $C_{h}$ in $\mathbb{C P}^{2}$ defined by

$$
\sum_{k=2}^{n} z^{n-k} H_{k}(x, y)+h z^{n}=0
$$

and let $\Sigma_{h}$ be the set of singularities of $\bar{C}_{h}$. Then there is a standard resolution of $\bar{C}_{h}$

$$
\phi_{h}: S_{h} \rightarrow \bar{C}_{h},
$$

where $S_{h}$ is the Riemann surface of $\bar{C}_{h}$ which is compact and connected, and $\phi_{h}$ is a holomorphic map and biholomorphic in $\phi_{h}^{-1}\left(\bar{C}_{h}-\Sigma_{h}\right)$. For any point $P \in \bar{C}_{h}-\Sigma_{h}$, there is only one point in $\phi_{h}^{-1}(P)$; while for $P \in \Sigma_{h}$, there may be more than one point in $\phi_{h}^{-1}(P)$. In fact, the number of points 
in $\phi_{h}^{-1}(P)$ is equal to the number of essentially different Puiseux expressions of $\bar{C}_{h}$ near the point $P$.

Note that the homogeneous part $H_{n}(x, y)$ of degree $n$ of $H(x, y)$ can be expressed as

$H_{n}(x, y)=\prod_{i=1}^{N}\left(\alpha_{i} x-\beta_{i} y\right)^{n_{i}}, \quad n_{i} \geq 1, \quad \sum_{i=1}^{N} n_{i}=n$,

where $\alpha_{i}, \beta_{i} \in \mathbb{C}$ such that $\alpha_{i}: \beta_{i} \neq \alpha_{j}: \beta_{j}$ if $i \neq j$. Then $\left[\beta_{i}: \alpha_{i}: 0\right]$ is the projective coordinate of a point $P_{h}^{i}$ at infinity on $C_{h}$. Denote by $P_{h}=\left\{P_{h}^{i}, i=1, \ldots, N\right\}, \tilde{P}_{h}^{i}=\phi_{h}^{-1}\left(P_{h}^{i}\right)$ and $\tilde{P}_{h}=\phi_{h}^{-1}\left(P_{h}\right)=\bigcup_{i=1}^{N} \tilde{P}_{h}^{i}$. If $\tilde{P}_{h}^{i}$ consists of $m_{i}$ points $\left\{\tilde{P}_{h}^{i 1}, \ldots, \tilde{P}_{h}^{i m_{i}}\right\}$, then in a sufficiently small neighborhood of each $\tilde{P}_{h}^{i j}$, we can associate a small loop $\tilde{\delta}_{h}^{i j} \in S_{h}-\tilde{P}_{h}$ centered at $\tilde{P}_{h}^{i j}$ at given orientation. Let $\delta_{h}^{i j}=\phi_{h}\left(\tilde{\delta}_{h}^{i j}\right)$.

In the one-dimensional homology group $\mathcal{H}_{1}\left(S_{h}-\tilde{P}_{h}, \mathbb{Z}\right)$, we can define some subgroups as follows: $\mathcal{E}_{h}$ is generated by $\left\{\tilde{\delta}_{h}^{i j}\right\}$ for all $i$ and $j$, and $\mathcal{E}_{h}^{12}$ is generated by all of those loops $\left\{\tilde{\delta}_{h}^{i j}\right\}$ such that each of them is centered at a point $\tilde{P}_{h}^{i j}$ of the first type or of the second type (see Definitions 2.3 and 2.4 below). Then for the corresponding vanishing cycle $\gamma_{h}$ of the origin, we have the following theorem.

Theorem 1. If the origin is an isochronous cen-

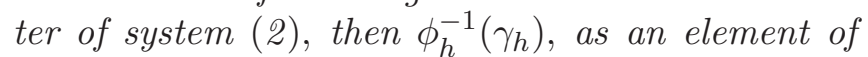
$\mathcal{H}_{1}\left(S_{h}-\tilde{P}_{h}, \mathbb{Z}\right)$, cannot belong to $\mathcal{E}_{h}^{12}$.

By this theorem, we have the following

Corollary 1.1. If the origin is an isochronous center of system (可) and $\mathcal{E}_{h}^{12}=\mathcal{E}_{h}$, then $\phi_{h}^{-1}\left(\gamma_{h}\right)$, as an element of the homology group $\mathcal{H}_{1}\left(S_{h}, \mathbb{Z}\right)$ of $S_{h}$, cannot be zero cycle.

Using Theorem 1 we will study the topology of an isochronous center for a large class of Hamiltonian systems with some $n_{i} \leq n / 2$ in the homogeneous part of degree $n$. Let $\mathcal{E}_{h}^{n / 2}$ represent a subgroup of $\mathcal{H}_{1}\left(S_{h}-\tilde{P}_{h}, \mathbb{Z}\right)$ generated by all of those $\left\{\tilde{\delta}_{h}^{i j}\right\}$ such that each of them is centered at a point $\tilde{P}_{h}^{i j}$ with $n_{i} \leq n / 2$ satisfying the NRF-condition (see Definition 2.2 below). Clearly $\mathcal{E}_{h}^{n / 2} \subset \mathcal{E}_{h}$. Then we have

Theorem 2. $\mathcal{E}_{h}^{n / 2} \subset \mathcal{E}_{h}^{12} \subset \mathcal{E}_{h}$.
By the above two theorems, we can immediately obtain the following

Corollary 1.2. If the origin is an isochronous center of system (2), then $\phi_{h}^{-1}\left(\gamma_{h}\right)$, as an element of $\mathcal{H}_{1}\left(S_{h}-\tilde{P}_{h}, \mathbb{Z}\right)$, cannot belong to $\mathcal{E}_{h}^{n / 2} ;$ furthermore, if $\mathcal{E}_{h}^{n / 2}=\mathcal{E}_{h}$ also holds, then $\phi_{h}^{-1}\left(\gamma_{h}\right)$, as an element of the homology group $\mathcal{H}_{1}\left(S_{h}, \mathbb{Z}\right)$ of $S_{h}$, cannot be zero cycle.

In reference Gavrilov, 1997], the author proved that if $H(x, y)$ is a good polynomial having only simple singularities (classified according to the Coxeter groups $A_{k}, D_{k}, E_{6}, E_{7}$ and $E_{8}$ (see [Arnold et al., 1985])), and the origin is a single critical point of Morse type which is isochronous on $C_{0}$, then the corresponding vanishing cycle represents a zero homology cycle on the Riemann surface of $C_{h}$. The precise definition of a good polynomial is given shortly below. According to this fact, one can immediately obtain a criterion for the nonisochronicity of a singularity of Morse type.

Corollary 1.3. For system (2), if $H(x, y)$ is a good polynomial having only simple singularities and containing a single critical point of Morse type in its critical level set of $H(x, y)=0$, and $\mathcal{E}_{h}^{12}=\mathcal{E}_{h}$, then the origin is not an isochronous center.

In the last section, we study the nonisochronicity for some classes of Hamiltonian systems using the above theorems and corollaries. In those examples, it is shown that our results can easily deal with the nonisochronicity of a singularity of Morse type for a number of Hamiltonian systems, especially for some systems having complicated forms which are very hard to handle by the classical tools such as normal form theory. Below, let us first provide some preliminaries such as definitions, notation and important lemmas, then give the detailed proof of the main results.

\section{Preliminaries}

First of all, we recall some basic facts from the theory of complex algebraic curves. For details, we recommend the readers to refer to Gavrilov, 1997; Kirwan, 1992; Milnor, 1968.

Given an irreducible polynomial $F(x, y)=$ $\sum_{k, l} a_{k l} x^{k} y^{l}$ in the polynomial ring $\mathbb{C}[x, y]$ with $F(0,0)=0$, denote by $\Lambda(F)$ the carrier of $F(x, y)$, 
i.e.

$$
\Lambda(F)=\left\{(k, l) \in \mathbb{Z}^{2}: a_{k l} \neq 0\right\} .
$$

Definition 2.1 [Newton Polygon]. If $Q_{1}, Q_{2} \in \mathbb{R}^{2}$, let

$$
\left[Q_{1}, Q_{2}\right]=\left\{s Q_{1}+(1-s) Q_{2}: 0 \leq s \leq 1\right\}
$$

be the straight line segment from $Q_{1}$ to $Q_{2}$. Consider the convex subset of $\mathbb{R}^{2}$ consisting of those $(x, y) \in \mathbb{R}^{2}$ such that $x \geq a$ and $y \geq b$ for some $(a, b) \in\left[Q_{1}, Q_{2}\right]$ where $Q_{1}$ and $Q_{2}$ belong to the carrier $\Lambda(F)$. Its boundary consists of a vertical half-line and a horizontal half-line joined by a union of finitely many straight line segments. This union is called the Newton polygon of $F(x, y)$ at $(x, y)=(0,0)$.

For any point $\tilde{P} \in S_{h}$, there is an essentially unique Puiseux expression parameterizing one of the components of $\bar{C}_{h}$ near $P=\phi_{h}(\tilde{P})$. For a point $P_{h}^{i} \in P_{h}$, up to a linear change of coordinates, we can always assume its projective coordinate is [1: $0: 0]$. To obtain the corresponding Puiseux expressions near $P_{h}^{i}$, it is convenient to adopt the coordinates $(u, v)$, where

$$
u=\frac{1}{x}, \quad v=\frac{y}{x} .
$$

Then the Puiseux expressions near $P_{h}^{i}$ are totally determined by the Puiseux expressions of $H_{h}^{*}(u, v)$ near the origin, where

$$
H_{h}^{*}(u, v)=u^{n} H\left(\frac{1}{u}, \frac{v}{u}\right)-h u^{n}=0 .
$$

According to the theory of Puiseux series, the following lemma holds.

Lemma 1 [Puiseux]. If $H_{h}^{*}(0,0)=0$ and $H_{h}^{*}(0$, $v) \neq 0$, then there exist numbers $p, q \in \mathbb{Z}_{+}$, a parameter $s \in \mathbb{C}$, and a holomorphic function $\rho(s)=$ $s^{q}\left(c_{0}+\sum_{i=1}^{+\infty} c_{i} s^{i}\right), c_{0} \neq 0$, such that $H_{h}^{*}\left(s^{p}, \rho(s)\right)=$ 0 for all $s$ in a neighborhood of 0 .

Since there are only finitely many essentially different Puiseux expressions, we can assume $p$ and $q$ have been chosen to be the minimum integers for each class. Let $\left\{\left(k_{i}, l_{i}\right), i=1, \ldots, m\right\}$ be the vertex set of the Newton polygon of $H_{h}^{*}(u, v)$ near the origin, where $l_{0} \geq l_{1} \geq \cdots \geq l_{m}=0,0=k_{0} \leq$ $k_{1} \leq \cdots \leq k_{m}$. Denoting by $\Delta=\min \left\{p k_{i}+q l_{i}, i=\right.$ $0, \ldots, m\}$, the minimum of $p k_{i}+q l_{i}$, there exists a straight line on $(k, l)$-plane

$$
L: p k+q l=\Delta
$$

passing all the points contained in $\Delta$, then one can define the Newton principal polynomial $g_{\Delta}(u, v)$ by the following

$$
g_{\Delta}(u, v)=\sum_{(k, l) \in L} b_{k, l} u^{k} v^{l}
$$

where we assume $H_{h}^{*}(u, v)=\sum_{k+l \geq 1} b_{k, l} u^{k} v^{l}$.

Definition 2.2 [NRF-Condition]. We say a point $\tilde{P}_{h}^{i j} \in \tilde{P}_{h}$ satisfies the NRF-condition, if the corresponding $g_{\Delta}(u, v)$ has no repeated factors except $u$ and $v$, where $g_{\Delta}(u, v)$ is defined as above by the corresponding Puiseux expression of $H_{h}^{*}(u, v)$ near the origin.

Definition 2.3 [The First Type]. We say a point $\tilde{P}_{h}^{i j} \in \tilde{P}_{h}$ is of the first type, if the corresponding $H_{h}^{*}(u, v)$ satisfies one of the following conditions near the origin:

(1) $q>2 p$;

(2) $q \leq 2 p<n p+q-\Delta$, and $\tilde{P}_{h}^{i j}$ satisfies the NRFcondition.

Definition 2.4 [The Second Type]. We say a point $\tilde{P}_{h}^{i j} \in \tilde{P}_{h}$ is of the second type, if the Newton polygon of the corresponding $H_{h}^{*}(u, v)$ near the origin consists of only one line segment containing points $(n, 0)$ and $(0, n / 2)$, where $n$ is even.

For each isolated critical point $P \in \mathbb{C}^{2}$ of $F(x, y)=0$, its Milnor number $\mu_{P}(F)$ is well defined by

$$
\mu_{P}(F)=\operatorname{dim}_{\mathbb{C}} \frac{\mathcal{O}_{P}(x, y)}{\left\langle F_{x}, F_{y}\right\rangle}
$$

where $\mathcal{O}_{P}(x, y)$ is the local ring of $\mathbb{C}^{2}$ at $P, F_{x}$ and $F_{u}$ are partial derivatives of $F(x, y)$. In Gavrilov, 1997], another topologically invariant $\lambda$ is well defined by the Milnor numbers of points at infinity as follows. For any $P \in \bar{C}_{h}-C_{h}$, let $\mu\left(\bar{C}_{h}, P\right)$ be the Milnor number of the germ of the analytic curve $\bar{C}_{h}$ at $P$, then for $h$ sufficiently close to $h_{0}$ but $h \neq h_{0}$, the number $\lambda_{P}^{h_{0}}(H)=\mu\left(\bar{C}_{h}, P\right)-\mu\left(\bar{C}_{h_{0}}, P\right)$ is well defined. The number $\lambda(H)$ is defined by

$$
\lambda(H)=\sum_{h \in \mathbb{C}_{P}} \sum_{P \in \bar{C}_{h}-C_{h}} \lambda_{P}^{h}(H) .
$$


Definition 2.5 [Good Polynomial]. Polynomial and $H(x, y)$ is said to be good if $\lambda(H)=0$.

\section{Proof of Theorems and Corollaries}

We first give the following two useful lemmas.

Lemma 2. If $\tilde{P}_{h}^{i j}$ is of the first type, then the integral $I_{i j}(h)=\oint_{\delta_{h}^{i j}} y \mathrm{~d} x$ is equal to a constant independent of $h$.

Proof. Without loss of generality, let $P_{h}^{i}=[1: 0$ : $0]$, then the corresponding

$$
\begin{aligned}
& H_{h}^{*}(u, v) \\
& =u^{n} H\left(\frac{1}{u}, \frac{v}{u}\right) \\
& \quad=\sum_{l=n_{i}}^{n} a_{n-l, l} v^{l}+\sum_{k=1}^{n-2} u^{k} \sum_{l=0}^{n-k} a_{n-k-l, l} v^{l}-h u^{n} \\
& =0,
\end{aligned}
$$

where $a_{n-n_{i}, n_{i}} \neq 0$. Assume that the Puiseux parameterization associated to $\tilde{P}_{h}^{i j}$ is

$$
u=s^{p}, \quad v=s^{q}\left(c_{0}+\sum_{i=1}^{+\infty} c_{i} s^{i}\right), \quad c_{0} \neq 0,
$$

where $p, q \in \mathbb{Z}_{+}$have been chosen to be minimum. Then the integral

$$
\begin{aligned}
I_{i j}(h) & =\oint_{\delta_{h}^{i j}} y \mathrm{~d} x=2 \pi \mathrm{i} \operatorname{Res}(y \mathrm{~d} x) \\
& =2 \pi \mathrm{i} \operatorname{Res}\left(-\frac{p s^{q}\left(c_{0}+c_{1} s+\cdots\right)}{s^{2 p+1}} \mathrm{~d} s\right) \\
& =-2 p \pi \mathrm{i} c_{2 p-q},
\end{aligned}
$$

where $\operatorname{Res}(\cdot)$ represents the residue of a meromorphic differential form at a pole.

If $2 p<q$, then $c_{2 p-q}=0$. If $2 p \geq q$, noticing that $\tilde{P}_{h}^{i j}$ is of the first type, then $q \leq 2 p<n p+$ $q-\Delta$ holds and $\tilde{P}_{h}^{i j}$ satisfies the NRF-condition. Comparing the coefficients of $s^{\Delta}$ and $s^{\Delta+2 p-q}$ on two sides of equation $H_{h}^{*}(u(s), v(s))=0$, we can obtain recursively

$$
g_{\Delta}\left(1, c_{0}\right)=\sum_{(k, l) \in L} a_{n-k-l, l} c_{0}^{l}=0
$$

$$
\begin{gathered}
\left(\sum_{(k, l) \in L} l a_{n-k-l, l} c_{0}^{l}\right) c_{2 p-q} \\
=\left(\left.c_{0} \frac{\partial g_{\Delta}(u, v)}{\partial v}\right|_{\left(1, c_{0}\right)}\right) c_{2 p-q} \\
=\tau\left(c_{0}, \ldots, c_{2 p-q-1}\right),
\end{gathered}
$$

where $\tau\left(c_{0}, \ldots, c_{2 p-q-1}\right)$ is a function in $c_{0}, \ldots$, $c_{2 p-q-1}$ which are independent of $h$. Note that $g_{\Delta}(u, v)$ has no repeated factors except $u$ and $v$, so the following holds:

$$
\sum_{(k, l) \in L} l a_{n-k-l, l} c_{0}^{l}=\left.c_{0} \frac{\partial g_{\Delta}(u, v)}{\partial v}\right|_{\left(1, c_{0}\right)} \neq 0,
$$

which implies $c_{2 p-q}$ is also independent of $h$.

Lemma 3. If $\tilde{P}_{h}^{i j}$ is of the second type, then the integral $I_{i j}(h)=\oint_{\delta_{h}^{i j}} y \mathrm{~d} x$ satisfies the following algebraic equation in $I_{i j}, h$,

$$
I_{i j}^{\frac{n}{2}}+b_{\frac{n}{2}-1} I_{i j}^{\frac{n}{2}-1}+\cdots+b_{1} I_{i j}+b_{0} h=0,
$$

where $b_{j}$ is a constant independent of $h$ for $j=$ $0,1, \ldots, n / 2-1$, and $b_{0} \neq 0$.

Proof. Perform the similar Puiseux parameterization to Lemma 2. Clearly in this case, $q=2 p$ and $c_{2 p-q}=c_{0}$. Comparing the coefficients of two sides of equation $H_{h}^{*}(u(s), v(s))=0$, it is not difficult to see that $c_{0}$ satisfies

$$
\begin{aligned}
g_{\Delta}\left(1, c_{0}\right) & =a_{\frac{n}{2}, \frac{n}{2}} c_{0}^{\frac{n}{2}}+\sum_{(k, l) \in L, k>0, l>0} a_{n-k-l, l} c_{0}^{l}-h \\
& =0 .
\end{aligned}
$$

By the assumption that $(n, 0)$ and $(0, n / 2)$ both belong to $L$, we have $a_{\frac{n}{2}, \frac{n}{2}} \neq 0$. Then the lemma obviously holds since $I_{i j}(h)=-2 p \pi \mathrm{i} c_{0}$.

Now we can prove Theorem 1 .

Proof [Proof of Theorem 1]. We suppose otherwise, that is, suppose $\phi_{h}^{-1}\left(\gamma_{h}\right) \in \mathcal{E}_{h}^{12}$, then it can be written as $\phi_{h}^{-1}\left(\gamma_{h}\right)=\sum_{i, j} d_{i j} \tilde{\delta}_{h}^{i j}$, where $d_{i j} \in \mathbb{Z}$, $\tilde{\delta}_{h}^{i j} \in \mathcal{E}_{h}^{12}$. 
According to the Gelfand-Leray derivative, the period function $T(h)=\oint_{\gamma_{h}} \mathrm{~d} t$ can be expressed as the derivative of an Abelian integral $I(h)=\oint_{\gamma_{h}} y \mathrm{~d} x$, i.e.

$$
T(h)=\oint_{\gamma_{h}} \mathrm{~d} t=\oint_{\gamma_{h}} \frac{1}{\frac{\partial H}{\partial y}} \mathrm{~d} x=I^{\prime}(h) .
$$

Denoting by $I_{i j}(h)=\oint_{\delta_{h}^{i j}} y \mathrm{~d} x$, then

$$
I(h)=\oint_{\gamma_{h}} y \mathrm{~d} x=\sum_{i, j} d_{i j} \oint_{\delta_{h}^{i j}} y \mathrm{~d} x=\sum_{i, j} d_{i j} I_{i j}(h) .
$$

Since the origin is isochronous, then $I^{\prime}(h)=T(h)$ is a constant independent of $h$. Assume $I(h)=A h+$ $B, A \neq 0$.

If $\tilde{P}_{h}^{i j}$ is of the first type, then, by Lemma 2 , $I_{i j}(h)$ is a constant independent of $h$. If $\tilde{P}_{h}^{i j}$ is of the second type, then, by Lemma $3 I_{i j}(h)$ satisfies an equation of the form (6), so it can be extended as a multivalued function with $n / 2$ single-valued components $I_{i 1}^{*}(h), \ldots, I_{i \frac{n}{2}}^{*}(h)$. Note that the left side of Eq. (6) is irreducible and has a ramification point with index $n / 2-1$ at $h=\infty$, thus a simple closed curve $h=\xi(\theta)=\operatorname{Re}^{\mathrm{i} \theta}$, where $\theta \in[0,2 \pi]$ and $R \in \mathbb{R}$ is sufficiently large, induces a permutation between $n / 2$ single-valued components of $I_{i j}(h)$ as roots of Eq. (6). Without loss of generality, we assume $I_{i j}\left(\operatorname{Re}^{\mathrm{i}(\theta+2(k-1) \pi)}\right)=I_{i k}^{*}\left(\operatorname{Re}^{\mathrm{i} \theta}\right)$, then by the relation between the roots and coefficients of Eq. (6), we have

$$
\sum_{k=1}^{n / 2} I_{i j}\left(\operatorname{Re}^{\mathrm{i}(\theta+2(k-1) \pi)}\right)=\sum_{k=1}^{n / 2} I_{i k}^{*}\left(\operatorname{Re}^{\mathrm{i} \theta}\right)=-b_{\frac{n}{2}-1},
$$

which is a constant independent of $h$. This leads to $\sum_{k=1}^{n / 2} I\left(\operatorname{Re}^{\mathrm{i}(\theta+2(k-1) \pi)}\right)$ and is also a constant independent of $h$. However, we already have

$$
\begin{aligned}
\sum_{k=1}^{n / 2} I\left(\operatorname{Re}^{\mathrm{i}(\theta+2(k-1) \pi)}\right) & =\sum_{k=1}^{n / 2} A \operatorname{Re}^{\mathrm{i}(\theta+2(k-1) \pi)}+B \\
& =\frac{n}{2}(A h+B), \quad A \neq 0,
\end{aligned}
$$

which deduces a contradiction.

Proof [Proof of Theorem 2]. Note that $\mathcal{E}_{h}^{12} \subset \mathcal{E}_{h}$ holds obviously. To prove $\mathcal{E}_{h}^{n / 2} \subset \mathcal{E}_{h}^{12}$, it suffices to show the point $\tilde{P}_{h}^{i j}$ such that the corresponding $\tilde{\delta}_{h}^{i j} \in \mathcal{E}_{h}^{n / 2}$ is either of the first type or of the second type.

We also assume $P_{h}^{i}=[1: 0: 0]$ and perform the Puiseux parameterization (5). If $q>2 p$ or $q \leq$ $2 p<n p+q-\Delta$, then $\tilde{P}_{h}^{i j}$ is of the first type. Below we assume $2 p \geq q$ and $2 p \geq n p+q-\Delta$. Taking a point $Q_{i}=\left(k_{i}, l_{i}\right) \in L$, we have $l_{i} \geq 1$ and $p k_{i}+$ $q l_{i}+2 p-q \geq n p$, which imply

$$
k_{i}+2 l_{i} \geq n .
$$

Denote by $L_{1}$ and $L_{2}$ the following lines respectively:

$$
\begin{aligned}
& L_{1}: k+2 l=k_{i}+2 l_{i}, \\
& L_{2}: k+2 l=n .
\end{aligned}
$$

Comparing positions of these lines, we have when $k \in\left[0, k_{i}\right]$, there are $L, L_{1}$ and $L_{2}$ from top to bottom. In addition, $(0, n / 2) \in L_{2}$ and $n_{i} \leq n / 2$ imply that the point $\left(0, n_{i}\right)$ cannot lie above $L$. However, $\left(0, n_{i}\right)$ is the left endpoint of Newton polygon of $H_{h}^{*}(u, v)$ near the origin, which cannot be below $L$, thus the only possible thing is $\left(0, n_{i}\right) \in L$, which results in $L=L_{2}$ and $n_{i}=n / 2$, i.e. $n$ is even, $q=2 p$ and $c_{2 p-q}=c_{0}$. Therefore $\tilde{P}_{h}^{i j}$ is of the second type. See Fig. 1 for the carrier and the Newton polygon of $H_{h}^{*}(u, v)$ in this case.

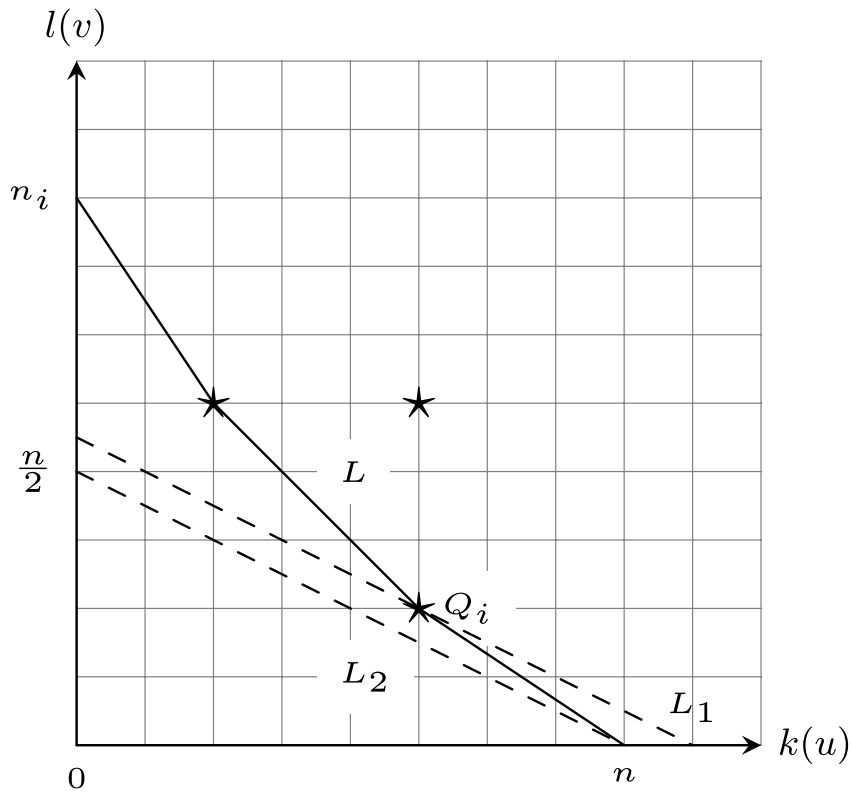

Fig. 1. Case $2 p \geq n p+q-\Delta$. 
Proof [Proof of Corollaries 1.1] and 1.2. They are the direct conclusions of Theorems 1 and 2, just noticing that $\mathcal{E}_{h}$ is trivial if we regard it as a subgroup of $\mathcal{H}_{1}\left(S_{h}, \mathbb{Z}\right)$.

Proof [Proof of Corollary 1.3]. Suppose that the origin is an isochronous center, then, by Theorem 4.1 in Gavrilov, 1997], $\phi_{h}^{-1}\left(\gamma_{h}\right)$ is the zero cycle of $\mathcal{H}_{1}\left(S_{h}, \mathbb{Z}\right)$, however, this contradicts Corollary 1.1.

\section{Applications}

Our theorems and corollaries provide us some criterions to judge the nonisochronicity of a center for Hamiltonian systems.

Example 4.1. Consider a system (2) with Hamiltonian $H(x, y)=x^{2} f(x)+y^{2} g(y)+a x^{n} y^{n}$, where $n>1, a \neq 0$ and $f(x)$ (resp., $g(y)$ ) is a polynomial of degree $k<2 n-2$ in one variable such that it has only simple roots and $f(0)=1$ (resp., $g(0)=1$ ). Then the origin is not an isochronous center.

In fact for any $k$ and $n$, the origin is the only critical point of Morse type on $H=0$, and $H$ has only simple singularities since the Hessian matrix of $H$ is not equal to 0 at any singularity. Besides, for each point at infinity the NRF-condition is satisfied and $\lambda(H)=0$, so $\mathcal{E}_{h}=\mathcal{E}_{h}^{12}$ and $H$ is good. Then, by Corollary 1.3 the origin is not isochronous.

Example 4.2. Consider a system (1) with Hamiltonian $H(x, y)=\left(x^{2}+y^{2}\right) / 2+H_{n}(x, y) \in \mathbb{R}[x, y]$, where $H_{n}(x, y)$ is a homogeneous polynomial of degree $n>2$.

In reference [Gasull et al., 1997], the authors provided a proof to show that there is no isochronous center for all of these systems. By Corollary 1.3, we can present another proof for the case when $H_{n}(x, y)$ has no factor $x^{2}+y^{2}$, which is usually considered as one of the most complicated cases in the sense of normal forms.

Regarding $H(x, y)$ as a polynomial in $\mathbb{C}[x, y]$, we have the following

- Assertion: $\mathcal{E}_{h}=\mathcal{E}_{h}^{12}$.

Proof [Proof of Assertion]. Noticing that up to a linear change of coordinates, we can assume $H=$ $a X^{2}+b X Y+c Y^{2}+Y^{n_{1}} f(X, Y)$, where $f(X, Y)$ has no factor $Y$.
If $n_{1}>n / 2$, then $Y$ is essentially a factor of $H_{n}(x, y)$ in $\mathbb{R}[x, y]$, this implies $a \neq 0$. In $(u, v)$-coordinate system as before, the corresponding Newton polygon of $H_{h}^{*}(u, v)$ contains only two points $\left(0, n_{i}\right)$ and $(n-2,0)$, thus the NRF-condition is satisfied and $p / q=n_{i} /(n-2)$. By the direct calculation, we have $\Delta=p(n-2)$ and $q \leq 2 p<$ $n p+q-\Delta$, that is, each point in $\phi_{h}^{-1}([1: 0: 0])$ is of the first type.

If $n_{1} \leq n / 2$, then $a$ and $b$ cannot be zero simultaneously. When $a \neq 0$, the NRF-condition is satisfied by the similar analysis as above. When $a=0$, the NRF-condition also holds unless the corresponding Newton polygon is one line segment containing three points $\left(0, n_{i}\right),(n-2,1)$ and $(n, 2)$, which is just the case when the point is of the second type. Then by Theorem 2, the assertion is proved.

If $H_{n}(x, y)$ has no factor $x^{2}+y^{2}$, then the origin is the only singularity on $H=0$ and is of Morse type. In addition, $H$ has only simple singularities since the Hessian matrix of $H$ is not equal to 0 at any singularity. For any $h \in \mathbb{C}$ and $P \in \bar{C}_{h}-C_{h}$, the number $\lambda_{P}^{h}(H)=0$, so $\lambda(H)=0$, i.e. $H$ is good. Therefore, by the above assertion and Corollary 1.3, the origin is not isochronous.

\section{Acknowledgments}

We are very grateful to the referees for their valuable suggestions to improve this paper. G. Dong is supported by NSFC 11701217 of China, NSF 2017A030310181 of Guangdong province (China), and Research Fund 21616312 of Jinan University (China). C. Liu and J. Yang are supported respectively by NSFC 11771315 and NSFC 11671016 of China.

\section{References}

Arnold, V. I., Gusein-Zade, S. M. \& Varchenko, A. N. [1985] Singularities of Differentiable Maps, Monographs in Mathematics, Vol. 82 (Birkhäuser, Boston).

Chavarriga, J. \& Sabatini, M. [1999] "A survey of isochronous centers," Qual. Th. Dyn. Syst. 1, 1-70.

Chen, X., Romanovski, V. G. \& Zhang, W. [2008] "Nonisochronicity of the center at the origin in polynomial Hamiltonian systems with even degree nonlinearities," Nonlin. Anal.: Th. Meth. Appl. 68, 2769-2778.

Chicone, C. \& Jacobs, M. [1989] "Bifurcation of critical periods," Trans. Amer. Math. Soc. 312, 433-486. 
Christopher, C. J. \& Devlin, C. J. [1997] "Isochronous centres in planar polynomial systems," SIAM J. Math. Anal. 28, 162-177.

Cima, A., Mañosas, F. \& Villadelprat, J. [1999] "Isochronicity for several classes of Hamiltonian systems," J. Diff. Eqs. 157, 373-413.

Cima, A., Gasull, A. \& Mañosas, F. [2000] "Period function for a class of Hamiltonian systems," J. Diff. Eqs. 168, 180-199.

Fernandes, W., Romanovski, V. G., Sultanova, M. \& Tang, Y. [2017] "Isochronicity and linearizability of a planar cubic system," J. Math. Anal. Appl. 450, 795-813.

Gasull, A., Guillamon, A., Mañosa, V. \& Mañosas, F. [1997] "The period function for Hamiltonian systems with homogeneous nonlinearities," J. Diff. Eqs. 139, 237-260.

Gavrilov, L. [1997] "Isochronicity of plane polynomial Hamiltonian systems," Nonlinearity 10, 433-448.

Han, M. \& Romanovski, V. G. [2012] "Isochronicity and normal forms of polynomial systems of ODEs," $J$. Symb. Comput. 47, 1163-1174.

Jarque, X. \& Villadelprat, J. [2002] "Nonexistence of isochronous centers in planar polynomial Hamiltonian systems of degree four," J. Diff. Eqs. 180, 334-373.
Kirwan, F. [1992] Complex Algebraic Curves, London Mathematical Society, Student Text, Vol. 23 (Cambridge University Press, Cambridge).

Llibre, J. \& Valls, C. [2011] "Classification of the centers and their isochronicity for a class of polynomial differential systems of arbitrary degree," Adv. Math. 227, 472-493.

Llibre, J. \& Romanovski, V. G. [2015] "Isochronicity and linearizability of planar polynomial Hamiltonian systems," J. Diff. Eqs. 259, 1649-1662.

Loud, W. S. [1964] "Behaviour of the period of solutions of certain plane autonomous systems near centers," Contrib. Diff. Eqs. 3, 21-36.

Mañosas, F. \& Villadelprat, J. [2002] "Area-preserving normalizations for centers of planar Hamiltonian systems," J. Diff. Eqs. 179, 625-646.

Milnor, J. [1968] Singular Points of Complex Hypersurfaces, Ann. Math. Studies, Vol. 61 (Princeton University Press, Princeton, NJ).

Wang, Z., Chen, X. \& Zhang, W. [2010] "Nonisochronicity of the center in polynomial Hamiltonian systems," Nonlin. Anal.: Th. Meth. Appl. 73, 228243. 